\title{
Serum Lycopene Concentration and Prostate Cancer Risk: Results from the Prostate Cancer Prevention Trial
}

\author{
Alan R. Kristal, Dr. P.H ${ }^{1}$, Cathee Till, M.S ${ }^{1}$, Elizabeth A. Platz, $\mathrm{PhD}^{2}$, Xiaoling Song, $\mathrm{PhD}^{1}$, \\ Irena B. King, $\mathrm{PhD}^{3}$, Marian L. Neuhouser, $\mathrm{PhD}^{1}$, Christine B. Ambrosone, $\mathrm{PhD}^{4}$, and lan \\ M. Thompson, MD, PhD $^{5}$ \\ ${ }^{1}$ Cancer Prevention Program, Fred Hutchinson Cancer Research Center, Seattle, WA \\ 2 Department of Epidemiology, Johns Hopkins Bloomberg School of Public Health, Baltimore, MD \\ 21205 \\ ${ }^{3}$ Department of Medicine University of New Mexico, Albuquerque, NM \\ ${ }^{4}$ Department of Cancer Prevention and Population Sciences, Roswell Park Cancer Institute, \\ Buffalo, NY \\ ${ }^{5}$ Department of Urology, The University of Texas Health Sciences Center at San Antonio, San \\ Antonio, TX
}

\begin{abstract}
Background-Lycopene has been promoted for prostate cancer prevention, despite the inconsistency of scientific evidence.

Methods-This nested case-control study examined whether serum lycopene was associated with prostate cancer risk among participants in the Prostate Cancer Prevention Trial (PCPT), a placebocontrolled trial of finasteride for prostate cancer prevention. Presence or absence of cancer was determined by prostate biopsy, recommended during the trial due to elevated prostate specific antigen (PSA) level or abnormal digital rectal exam (DRE) and offered to all men at the trial end. There were 1683 cases ( 461 Gleason score $\geq 7,125$ Gleason score $\geq 8$ ) and 1751 controls.
\end{abstract}

Results-There were no associations of lycopene with prostate cancer risk. The odds ratios for a linear increase in lycopene (per 10ug/dl) were 0.99 [95\% CI 0.94-1.04], 1.01 [0.94-1.08] and 1.02 [0.90-1.15] for Gleason 2-6, 7-10, and 8-10, respectively. In the placebo arm, a 10ug/dl increase in lycopene was associated with a 7\% [95\% CI 14-0\%] reduced risk of cancer diagnosed following an elevated PSA or abnormal DRE, which are cancers that best match those detected in screened populations. However, a 10ug/dl increase in lycopene was also associated with an $8 \%$ [95\% CI 1$16 \%$ ] increased risk of cancer diagnosed without a biopsy prompt, which are cancers generally not detected. These findings were similar for low- and high-grade cancer.

Conclusion-This study does not support a role for lycopene in prostate cancer prevention.

Impact-Scientists and the public should understand that early studies supporting an association of dietary lycopene with reduced prostate cancer risk have not been replicated in studies using serum biomarkers of lycopene intake. Recommendations from professional societies to the public should be modified to reflect that the likelihood that increasing lycopene intake will not affect prostate cancer risk.

Corresponding Author Alan R. Kristal, Dr.P.H., Member and Associate Head, Cancer Prevention Program, Fred Hutchinson Cancer Research Center, 1100 Fairview Avenue North, M4-B402, PO Box 19024, Seattle, WA 98109-1024, Phone: (206) 667-4686, Fax: (206)667-7850, akristal@fhcrc.org. 


\section{Keywords}

Prostate cancer; biomarkers; nutrients; lycopene

\section{Introduction}

Whether or not high consumption of lycopene, a carotenoid found primarily in tomatoes and tomato products, reduces the risk of prostate cancer remains controversial $(1,2)$. This is an important question from a public health perspective, because increasing the consumption of lycopene through dietary change, food fortification or supplementation would be relatively simple and inexpensive interventions for prostate cancer prevention. Lacking a large, randomized clinical trial, the best evidence for an association of lycopene with prostate cancer will be based on large cohort studies; and, due to well established limitations of dietary assessment(3), somewhat stronger inferences can be made when using prediagnostic serum lycopene concentration rather than self-reported dietary intake as a measure of exposure. Eleven cohort studies have examined prediagnostic serum lycopene and prostate cancer risk(4-13). Of these, none reported statistically significant associations in unstratified analyses and three reported significant inverse associations in subgroups defined by age(4), family history of prostate cancer(4) and cancer aggressiveness $(6,11)$. There are also six cohort studies that have examined self-reported dietary lycopene and/or consumption of foods high in biologically-available lycopene such as tomato sauce or foods made with cooked tomatoes(14-21). Of these, one found significant inverse associations overall(20), which differed somewhat by tumor grade and stage(19), and one found an inverse association in a subgroup defined by family history of prostate cancer(17). Null findings from the majority of prospective studies of lycopene and prostate cancer risk could be attributed to many factors, including: small sample sizes, especially of advanced stage cancer; lycopene intakes too low to observe a possible threshold effect; and the widespread use of Prostate Specific Antigen (PSA) screening that has led to the diagnosis of many local stage and low grade tumors of little clinical importance(22). Nevertheless, additional studies that address methodological weaknesses in the previous research and confirm or refute previous subgroup findings are needed.

Here we give results of a study examining the associations of serum lycopene concentration with the risk of prostate cancer in a large cohort of men participating in the Prostate Cancer Prevention Trial (PCPT). Several aspects of the PCPT are unique, in particular the biopsydetermined absence or presence of cancer and the centralized and uniform pathological grading used to define cancer endpoints. Thus, while almost all prostate cancer cases were local stage and findings may not be generalizable to populations not subject to widespread PSA screening, detection bias was minimized and pathological grading of cases was rigorous and standardized. Furthermore, because blood samples were collected each year during the trial, it was feasible to pool plasma samples from multiple years to better characterize long-term lycopene exposure. Results from this study can help clarify whether, in the context of contemporary prostate cancer epidemiology, lycopene should be a candidate for further investigation as a cancer chemopreventive agent.

\section{Materials and Methods}

\section{Study Design and Study Population}

Data are from the PCPT, a randomized, placebo-controlled trial that tested whether finasteride, a $5 \alpha$-reductase inhibitor, could reduce the 7-year period prevalence of prostate cancer. Details regarding study design and participant characteristics have been described previously(23). Briefly, 18,880 men age 55 years and older with normal digital rectal exam 
(DRE) and PSA levels of $3 \mathrm{ng} / \mathrm{ml}$ or below, as well as no history of prostate cancer, severe lower urinary tract symptoms (defined as an International Prostate Symptom Score of 19 or lower) or clinically significant coexisting conditions (judged by the clinical site physician to affect survival or eligibility for the end-of-study biopsy at 7 years post-randomization), were randomized to receive finasteride ( $5 \mathrm{mg} /$ day) or placebo. During the PCPT, men underwent DRE and PSA determinations annually, and a prostate biopsy was recommended for participants with an abnormal DRE or if a PSA adjusted for the effect of finasteride was 4.0 $\mathrm{ng} / \mathrm{ml}$ or greater. At the final study visit at year 7 , all men not previously diagnosed with prostate cancer were requested to undergo an end-of-study prostate biopsy. All biopsies consisted of a minimum of 6 cores collected under transrectal ultrasonographic guidance and were reviewed for adenocarcinoma by both the pathologist at the local study site and a central pathology laboratory with concordance achieved in all cases. Clinical stage was assigned locally and tumors were graded centrally using the Gleason scoring system. Prostate cancer cases were classified as "for-cause" if there was a prompt for biopsy based on an abnormal DRE or elevated PSA, and "not-for-cause" if there was no prompt preceding the end-of-study biopsy. All men gave informed consent and study procedures were approved by Institutional Review Boards at each study center, the Southwest Oncology Group (SWOG, San Antonio, TX), and the Fred Hutchinson Cancer Research Center (Seattle, WA).

\section{Case and control selection}

The study reported here is from a large nested case-control study designed to examine multiple hypotheses about prostate cancer biology and risk $(24)$. Cases $(n=1,809)$ were all men with biopsy-confirmed cancer identified before study unblinding who had baseline blood samples available for analysis and controls $(n=1,809)$ were selected from men who were disease-free at the end-of-study biopsy and had baseline blood samples. Controls were frequency-matched to cases on distributions of age ( \pm 5 years), treatment group (finasteride or placebo), and a first-degree relative with prostate cancer, and were oversampled for nonwhites.

\section{Data Collection and Laboratory Methods}

Information on age, race, diabetes status, family history of prostate cancer in first-degree relatives and history of smoking was collected at baseline using self-administered questionnaires. Participants' height and weight were measured at baseline, and body mass index (BMI) was calculated as weight $(\mathrm{kg}) /$ height $\left(\mathrm{m}^{2}\right)$.

Non-fasting blood was collected approximately 3 months prior to randomization and annually thereafter until diagnosis or the end of the study. Venous blood was drawn into glass collection tubes without anticoagulant, refrigerated, and shipped to a central repository where they were centrifuged, aliquoted, and stored at $-70^{\circ} \mathrm{C}$. Lycopene concentration was measured in $0.5 \mathrm{ml}$ serum samples that were collected at years 1 and 4 , pooled and refrozen at $-70^{\circ} \mathrm{C}$ before analysis. Post-randomization bloods were used to conserve the limited prerandomization samples, and the two samples were pooled to reduce intra-individual variability. In a pilot study of 45 men from this cohort, the intraclass correlation for serum lycopene, based on samples from years 1, 5 and 7, was 0.76; the Spearman-Brown predicted reliability(25) of the mean of two samples used in this study was therefore 0.86 . Alternate years were selected if men were missing a year 1 or 4 sample or were diagnosed before year 4 ( $\mathrm{n}=320$ cases, 130 controls), a single sample was used if two prediagnostic blood samples were not available ( $\mathrm{n}=75$ cases, 3 controls), and men diagnosed with cancer before a postrandomization blood was collected were not eligible $(n=44)$. More cases were missing multiple post-diagnostic bloods because their participation in the trial ended at the time of diagnosis, whereas controls had to remain in the trial for 7 years. After further excluding 
men with insufficient serum ( $\mathrm{n}=22$ cases, 4 controls), men missing one or more covariates $(n=16$ cases, 19 controls) and men missing laboratory values due to a labeling error $(n=44$ cases, 35 controls), there were 1751 controls and 1683 cases available for this analysis.

Total lycopene concentration, specifically the sum of all lycopene isomers, was measured by high performance liquid chromatography as follows: A hexane extraction of serum, prepared under yellow light using tertiary butylhydroquinone as an antioxidant, was injected onto a 3um C-18 Spherisorb ODS-2 HPLC column (125×3 MM, Waters PSS838528) and eluted with an isocratic solvent consisting of $76 \%$ acetonitrile, $12 \%$ tetrahydrofuran, $5 \%$ methanol, $7 \%$ water, $0.025 \%$ ammonium acetate and $0.05 \%$ diethyl amine (v/v) at the flow rate of 0.7 $\mathrm{ml} /$ minute. Lycopene was detected at $476 \mathrm{~nm}$. Standard curves were generated with commercially available pure chemicals. The coefficient of variation for pooled quality control samples was $13.5 \%$. Total cholesterol was measured on Roche Cobas Mira Plus Chemistry Analyzer using the Roche cholesterol reagent (cat no 3313000, Roche Diagnostics). Assays were completed by the Fred Hutchinson Cancer Research Center Nutritional Biomarkers Laboratory, which participates in the National Institute of Standards and Technology Micronutrients Measurement Quality Assurance Program for fat-soluble vitamins and carotenoids in human plasma. The coefficient of variation for pooled quality control samples was $3.7 \%$. All batches were balanced for numbers of cases and controls.

\section{Statistical Analysis}

We used logistic and polytomous logistic models to estimate associations of serum lycopene concentration with risks of total, low-and high-grade disease. Low-grade was defined as Gleason score 2-6 and high-grade was classified as both Gleason score 7-10 and, more conservatively, as Gleason score 8-10. Results are given for finasteride and placebo arms separately, because we had hypothesized a priori that finasteride treatment could modify associations between risk factors and cancer; results are also given for both treatment arms combined. Models were adjusted for matching variables and variables associated with prostate cancer risk in this cohort, including age (continuous), race (Caucasian, other), family history of prostate cancer in first-degree relatives (yes, no), diabetes (yes, no), body mass index (continuous) and serum cholesterol (continuous). In analyses of the combined treatment arms, models were additionally controlled for treatment (finasteride, placebo). Further control for education, smoking, baseline PSA and physical activity did not affect results and are not included in final models. Lycopene was analyzed both categorized in quartiles, defined by the distribution in controls, and as a continuous variable (per 10ug/dl). Analyses were also stratified by age ( $<60 \mathrm{yrs}, 60-64 \mathrm{yrs}, 65-69 \mathrm{yrs}, \geq 70 \mathrm{yrs}$ ), race (AfricanAmerican, white), family history of prostate cancer (yes, no), and BMI ( $<25 \mathrm{~kg} / \mathrm{m}^{2}, 25-29$ $\mathrm{kg} / \mathrm{m}^{2}, \geq 30 \mathrm{~kg} / \mathrm{m}^{2}$ ), and whether or not the diagnostic biopsy was "for-cause" (following a PSA $>4 \mathrm{ng} / \mathrm{ml}$ or abnormal DRE). Due to small numbers, results from stratified analyses are given only for high-grade cancer defined as Gleason score 7-10; however, there were no substantive differences when high-grade was defined as Gleason score 8-10 or (4+3) plus 810. Tests for linear trend across quartiles were based on an ordinal variable corresponding to rank from lowest to highest category, as described by Breslow and Day (26). Tests for differences in associations across strata were based on interaction terms between serum lycopene trend (as described above) and categorical indicator variables for race and family history and ordinal variables corresponding to rank for age and BMI. A Wald chi-square test was used to evaluate whether the linear trend of lycopene with cancer risk differed between for-cause and not-for-cause cancers. All analyses were performed using SAS version 9.2 (SAS Institute Inc., Cary, North Carolina). 


\section{Results}

Table 1 gives demographic and health-related characteristics of the study population, stratified by presence or absence of prostate cancer and by grade. Due to the sampling design, there were more non-white controls than cases and no difference between cases and controls in age, family history of prostate cancer and treatment arm. Controls were more likely than cases to have diabetes, but there were no differences between cases and controls in BMI, smoking history or serum lycopene concentration. Almost $75 \%$ of cases were clinical stage T1,24\% were stage T2, and only $1.5 \%$ were stage $\mathrm{T} 3$. The proportion of cancer cases diagnosed for cause was much higher for high-compared to low-grade disease.

Table 2 gives associations of serum lycopene concentrations with prostate cancer risk. There were no significant associations for total, low- or high-grade cancer, in either the placebo or finasteride arms separately or in the study arms combined.

There were no significant associations within or differences between strata defined by age, race, BMI or family history of prostate cancer (data not shown). However, there were significant differences between cancers diagnosed for-cause and not-for-cause (Table 3 ). In the placebo arm, there were borderline statistically significant reductions in for-cause cancers of approximately $25 \%$ in quartile $2(\mathrm{Q} 2), \mathrm{Q} 3$ and Q4 compared to Q1, with no evidence of dose-response; the odds ratio for the a-posteriori contrast of Q2-Q4 vs. Q1 was 0.73 [95\% CI 0.56-0.95, p<0.02]. In contrast, increasing lycopene concentration was associated with a linear increase in the risk of cancer diagnosed not-for-cause; in the continuous model each $10 \mathrm{ug} / \mathrm{dl}$ increase was associated with an 8\% [95\% CI 1\%-16\%] increase in risk. Findings in the placebo arm were similar for low- and high-grade cancer, although the numbers of cases were small and no associations reached statistical significance. In the placebo arm, the associations of lycopene with for-cause and not-forcause cancers differed significantly for total and low-grade cancer $(\mathrm{p}<0.01, \mathrm{p}=0.01$, respectively). In the finasteride arm, increasing serum lycopene was associated with a significant, linear decrease in the risk of low-grade cancer only; each $10 \mathrm{ug} / \mathrm{dl}$ increase was associated with a $12 \%$ [95\% CI 22\%-0\%] reduced risk. There were no associations of lycopene with not-for-cause or high-grade cancers. In the finasteride arm, the associations of lycopene with for-cause and not-for-cause cancers differed significantly for low-grade cancer only $(\mathrm{p}=0.03)$. In the combined arms, each $10 \mathrm{ug} / \mathrm{dl}$ increase in serum lycopene was associated with a $6 \%$ [95\% CI 12\%-1\%] decrease and a 5\% [95\% CI -1\%-11\%] increase in the risks of for-cause and not-for-cause cancers, respectively. When stratified by grade, there was a significant inverse but non-linear association of lycopene with low-grade, forcause cancer, but no significant associations with not-for cause or high-grade cancers. In the total sample, the associations of lycopene with for-cause and not-for-cause cancers differed significantly for total and low-grade cancer only (both $\mathrm{p}<0.01$ ).

\section{Discussion}

In this study of primarily asymptomatic, local stage prostate cancer, pre-diagnostic serum lycopene concentration was not associated with the risk of total, low- or high-grade cancer. There were also no associations of serum lycopene with prostate cancer risk within strata defined by age, race, BMI or family history of prostate cancer. However, in the placebo arm of the trial, increasing serum lycopene was associated with reduced risk of prostate cancer that was diagnosed following either an elevated PSA test or abnormal DRE and a corresponding increased risk of cancer diagnosed at the end of the study without indication for biopsy. In the finasteride arm, increasing serum lycopene was associated only with decreased risk of low-grade cancer diagnosed for cause. 
The overall lack of association between serum lycopene concentration and prostate cancer risk found in this study is consistent with previous studies $(2,4-13)$, which found no significant associations in unstratified analyses. Comparing our findings to previouslyreported findings in subgroups requires careful evaluation of each. Two studies have reported significant inverse associations of lycopene with risk of high grade/advanced stage cancer only: In the placebo arm of the Physicians Health Study (PHS), a randomized trial of aspirin and $\beta$-carotene supplementation, there was a $60 \%$ reduction in risk of aggressive cancer comparing the highest to lowest quintiles of serum lycopene(6); and in the European Prospective Investigation into Cancer and Nutrition (EPIC) there was a $60 \%$ reduced risk of advanced prostate cancer among men in the highest compared to lowest quintile of plasma lycopene(11). There was a dose-response association in the PHS but an inverted J-shaped association in EPIC. Neither this nor any of the four previously-published studies that examined associations stratified by grade and/or stage have replicated these findings $(4,5,7,13)$. In the Health Professionals Follow-Up Study there were $63 \%$ and 52\% reduced risks among men who, respectively, provided their serum samples at age $\geq 65$ years and had no family history of prostate cancer(4). Neither this nor any of the four previouslypublished studies that examined associations stratified by age $(6,7,11,13)$ or the one study stratified family history of prostate cancer(13) replicated these findings. Our study provides no support of previous subgroup findings from serum-based studies, which with the exception of two studies finding associations for aggressive disease, have never been replicated.

Findings from cohort studies based on self-reported diet provide little additional clarity on subgroup findings in serum-based studies, specifically regarding whether associations of lycopene with prostate cancer risk are limited to older men, men with advanced disease or men without a family history of prostate cancer. In the PHS, high tomato sauce intake was associated with similar reductions in risk of organ-confined, minimally-extraprostatic and advanced disease, but larger reductions in risk for low- compared to high-grade disease(19). In the Prostate Lung Colorectal and Ovarian Cancer Screening Trial (PLCO) there was an inverse association of lycopene intake only among men with a family history of prostate cancer(17). Even within cohorts, no statistically significant subgroup finding has been consistent between diet- and serum-based analyses $(4,13,17,19)$. Given that both this study and the majority of previously-published cohort studies based on either serum or dietary measures of lycopene exposure have found no association either overall or in subgroups, and that a small number of subgroup findings are not consistent across or even within studies, we judge that the accumulated evidence does not support an association lycopene with prostate cancer.

This study found inverse associations of serum lycopene with cancer diagnosed for cause (following a biopsy prompt) and positive associations with cancer diagnosed not-for-cause (at the protocol-specified end of the study biopsy). Results were somewhat inconsistent across study arms; we therefore first consider the placebo arm, in which findings were similar for low-and high-grade cancer. Cancers diagnosed for-cause were more likely to be higher-grade and higher-volume (23), as both are associated with elevated PSA. We considered whether lycopene preferentially prevented more clinically-significant cancers, however this was unlikely because findings did not differ by grade. High lycopene concentration could have delayed or prevented cancer detection if serum lycopene or a factor association with serum lycopene was inversely associated with PSA. There was a weak inverse association of serum lycopene with PSA in the placebo but not finasteride arm: controlled for age, race, family history, BMI and cholesterol: $\beta$ (ng/ml PSA per $10 \mathrm{ug} / \mathrm{dl}$ lycopene $)=-0.041[95 \% \mathrm{CI}-0.013--0.069]$ and $-0.006[95 \% \mathrm{CI}-0.026-0.038]$ in the placebo and finasteride arms, respectively; $p_{\text {inter }}<0.03$. We know of no biological explanation for this finding, however it does suggest that when using current PSA screening 
practices high serum lycopene could delay or prevent prostate cancer diagnosis. We next considered findings in the finasteride arm, in which there was an inverse association of lycopene with low-grade, for-cause cancers only, with no association with high-grade or not-for-cause cancer. The lack of association with high-grade disease may be due to the increase in the sensitivities of both PSA screening and DRE to detect high-grade cancer among men receiving finasteride $(27,28)$, which could attenuate any association of lycopene on the detection of high-grade cancer. We have no hypotheses to explain the inverse association of lycopene with for-cause but not not-for-cause, low-grade cancer. It is also possible that all of these subgroup findings could be due to chance, reflecting the large number of subgroup analyses completed within these data. The clinical significance of these findings is uncertain. It would be beneficial if high lycopene intake delayed or prevented the detection of local stage, low-grade cancers that were of no clinical significance. In contrast, it would be harmful if high lycopene intake delayed or prevented detection of high-grade prostate cancer, as these are generally aggressive and far more likely to metastasize and cause death.

There are unique aspects to this study that must be considered when interpreting its results. Most importantly, study participants had PSA $<3 \mathrm{ng} / \mathrm{ml}$ at study entry and received annual screening (PSA plus DRE) during the 7 years of the trial and, further, almost half of the cancers were detected at the end-of-study biopsy among men without an elevated PSA or abnormal DRE. Therefore the incidence of cancer and the proportion of cancers that were low-grade and local-stage were higher in the PCPT than in other studies. We note that in the placebo arm, findings for for-cause cancers can be directly compared to studies in populations undergoing routine PSA screening. However, when considering the positive association with cancers detected by not-for-cause biopsy and the null findings for all cancers combined, it appears that the inverse associations found for screen-detected cancers were misleading. Lycopene assessment was from two samples collected approximately 3 years apart, which we believe is superior to measuring lycopene based on self-reported "usual" diet because the correlation between dietary lycopene as measured by the PCPT food frequency questionnaire (FFQ) and serum lycopene was low (0.12) and the effective reliability of the serum measure was higher (0.86) than the 6-month test-retest reliability of the PCPT FFQ (0.48). However, blood samples were not protected from light during collection and were subject to one freeze-thaw cycle in the preparation of the pooled aliquots; this will add error to the lycopene assay but there is no reason to believe that this error would be biased by case/control status.

A major strength of the PCPT is the mitigation of detection biases present in most observational cohorts in which PSA level and digital rectal examinations affect the decision to perform a prostate biopsy. Use of PSA screening is likely associated with dietary patterns(29), such that biases due to screening may have seriously confounded previous studies. An additional strength is the availability of Gleason score based on a single, research pathologist, in contrast to other studies that have classified the aggressiveness of incident cancers using a mix of clinical and pathological (post-prostatectomy) stage, grade that is either qualitative or assigned by multiple clinical pathologists, or long-term clinical outcomes. Finally, this study, with 1,683 (461 high-grade) cases was substantially larger than the 692 (235 high-grade) in the PLCO (13), which is the next largest study.

In conclusion, we found no evidence in this unique sample of primarily local stage, biopsydetected cancers that serum lycopene is associated with reduced prostate cancer risk. In men not treated with finasteride, lycopene was associated with delayed detection of both lowand high-grade cancers, which was an unexpected finding of uncertain clinical significance. Overall our findings are consistent with those from most other cohort studies, which taken together do not support the use of lycopene for the prevention of prostate cancer. 


\title{
Acknowledgments
}

\author{
Supported by grants: \\ P01 CA37429, Prostate Cancer Prevention Trial \\ P01 CA108964, Biology of the Prostate Cancer Prevention Trial
}

\section{References}

1. Kavanaugh CJ, Trumbo PR, Ellwood KC. The US Food and Drug Administration's Evidence-Based Review for Qualified Health Claims: Tomatoes, Lycopene, and Cancer. JNCI Journal of the National Cancer Institute. 2007; 99:1074-85.

2. Giovannucci E. Does prostate-specific antigen screening influence the results of studies of tomatoes, lycopene, and prostate cancer risk? J Natl Cancer Inst. 2007; 99:1060-2. [PubMed: 17623795]

3. Kristal AR, Peters U, Potter JD. Is it time to abandon the food frequency questionnaire? Cancer Epidemiol Biomarkers Prev. 2005; 14:2826-8. [PubMed: 16364996]

4. Wu K, Erdman JW, Schwartz SJ, et al. Plasma and the dietary carotenoids, and the risk of prostate cancer: a nested case-control study. Cancer Epidemiol Biomarkers Prev. 2004; 13:260-9. [PubMed: 14973107]

5. Gill JK, Franke AA, Steven Morris J, et al. Association of selenium, tocopherols, carotenoids, retinol, and 15-isoprostane $\mathrm{F} 2 \mathrm{t}$ in serum or urine with prostate cancer risk: the multiethnic cohort. Cancer Causes Control. 2009; 20:1161-71. [PubMed: 19212706]

6. Gann P, Ma J, Giovannucci E, et al. Lower prostate cancer risk in men with elevated plasma lycopene levels: results of a prospective analysis. Cancer Res. 1999; 59:1225-30. [PubMed: 10096552]

7. Huang H-Y, Alberg AJ, Norkus EP, Hoffman SC, Comstock GW, Helzlsouer KJ. Prospective study of antioxidant micronutrients in the blood and the risk of developing prostate cancer. Am $\mathrm{J}$ Epidemiol. 2003; 157:335-44. [PubMed: 12578804]

8. Karppi J, Kurl S, Nurmi T, Rissanen TH, Pukkala E, Nyyssonen K. Serum Lycopene and the Risk of Cancer: The Kuopio Ischaemic Heart Disease Risk Factor (KIHD) Study. Ann Epidemiol. 2009; 19:512-8. [PubMed: 19443241]

9. Beilby J, Ambrosini GL, Rossi E, de Klerk NH, Musk AW. Serum levels of folate, lycopene, carotene, retinol and vitamin E and prostate cancer risk. Eur J Clin Nutr. 2010 e-pub ahead of print.

10. Goodman GE, Schaffer S, Omenn GS, Chen C, King I. The association between lung and prostate cancer risk, and serum micronutrients: results and lessons learned from $\beta$-carotene and Retinol Efficacy Trial. Cancer Epidemiol Biomarkers Prev. 2003; 12:518-26. [PubMed: 12814997]

11. Key TJ, Appleby PN, Allen NE, et al. Plasma carotenoids, retinol, and tocopherols and the risk of prostate cancer in the European Prospective Investigation into Cancer and Nutrition study. Am J Clin Nutr. 2007; 86:672-81. [PubMed: 17823432]

12. Nomura AM, Stemmermann GN, Lee J, Craft NE. Serum micronutrients and prostate cancer in Japanese Americans in Hawaii. Cancer Epidemiol Biomarkers Prev. 1997; 6:487-91. [PubMed: 9232334]

13. Peters U, Leitzmann MF, Chatterjee N, et al. Serum lycopene, other carotenoids, and prostate cancer risk: a nested case-control study in the prostate, lung, colorectal, and ovarian cancer screening trial. Cancer Epidemiol Biomarkers Prev. 2007; 16:962-8. [PubMed: 17507623]

14. Schuurman A, Goldbohm RA, Brants HAM, van den Brandt PA. A prospective cohort study on intake of retinol, viatmins $\mathrm{C}$ and $\mathrm{E}$, and carotenoids and prostate cancer risk (Netherlands). Cancer Causes Control. 2002; 13:573-82. [PubMed: 12195647]

15. Kristal AR, Arnold KB, Neuhouser ML, et al. Diet, supplement use, and prostate cancer risk: results from the Prostate Cancer Prevention Trial. Am J Epidemiol. 2010; 172:566-77. [PubMed: 20693267]

16. Takachi R, Inoue M, Sawada N, et al. Fruits and vegetables in relation to prostate cancer in Japanese men: the Japan Public Health Center-Based Prospective Study. Nutr Cancer. 2010; 62:30-9. [PubMed: 20043257] 
17. Kirsh VA, Mayne ST, Peters U, et al. A prospective study of lycopene and tomato product intake and risk of prostate cancer. Cancer Epidemiol Biomarkers Prev. 2006; 15:92-8. [PubMed: 16434593]

18. Ambrosini GL, de Klerk NH, Fritschi L, Mackerras D, Musk B. Fruit, vegetable, vitamin A intakes, and prostate cancer risk. Prostate Cancer Prostatic Dis. 2008; 11:61-6. [PubMed: 17519926]

19. Giovannucci E, Liu Y, Platz EA, Stampfer MJ, Willett WC. Risk factors for prostate cancer incidence and progression in the Health Professionals Follow-Up Study. Int J Cancer. 2007:121. [PubMed: 17019706]

20. Giovannucci EL, Rimm EB, Liu Y, Stampfer MJ, Willett WC. A prospective study of tomato products, lycopene, and prostate cancer risk. J Natl Cancer Inst. 2002; 94:391-8. [PubMed: 11880478]

21. Stram D, Hankin J, Wilkens L, et al. Prostate cancer incidence and intake of fruits, vegetables and related micronutrients: the multiethnic cohort study*(United States). Cancer Causes Control. 2006; 17:1193-207. [PubMed: 17006725]

22. Cooperberg MT, Lubeck DP, Meng MV, Mehta SS, Carroll PR. The changing face of low-risk prostate cancer: trends in clinical presentation and primary management. J Clin Oncol. 2004; 22:2141-9. [PubMed: 15169800]

23. Thompson IM, Goodman PJ, Tangen CM, et al. The influence of finasteride on the development of prostate cancer. N Engl J Med. 2003; 349:215-24. [PubMed: 12824459]

24. Goodman, PJ.; Tangen, CM.; Kristal, AR., et al. Transition of a clinical trial into translational research: The Prostate Cancer Prevention Trial experience. Cancer Prevention Research; in press

25. White, E.; Armstrong, BK.; Saracci, R. Principles of exposure measurement in epidemiology. New York: Oxford University Press; 2008.

26. Breslow, NE.; Day, NE. The analysis of case-control studies. Vol. 1. Lyon: Intl Agency for Research on Cancer; 1980. Statistical methods in cancer research.

27. Thompson IM, Chi C, Ankerst DP, et al. Effect of finasteride on the sensitivity of PSA for detecting prostate cancer. J Natl Cancer Inst. 2006; 98:1128-33. [PubMed: 16912265]

28. Thompson IM, Tangen CM, Goodman PJ, et al. Finasteride improves the sensitivity of digital rectal examination for prostate cancer detection. J Urol. 2007; 177:1749-52. [PubMed: 17437804]

29. Close DR, Kristal AR, Patterson RE, White E. Associations of demographic and health-related characteristics with prostate cancer screening in Washington State. Cancer Epidemiol Biomarkers Prev. 1998; 7:627-30. [PubMed: 9681532] 


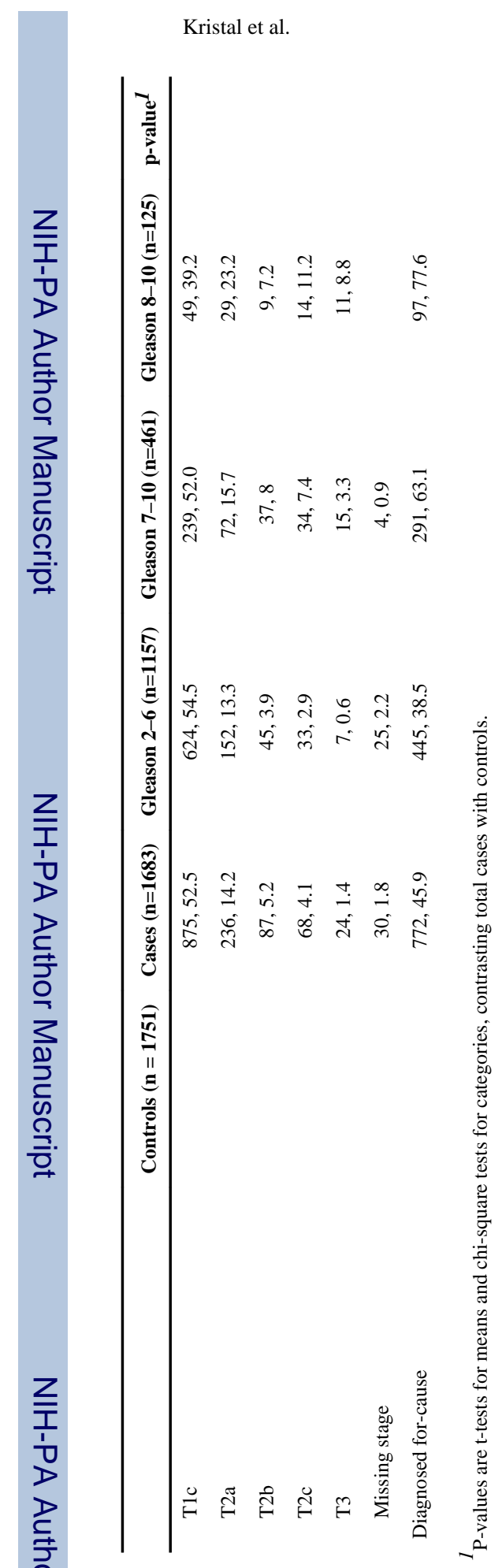




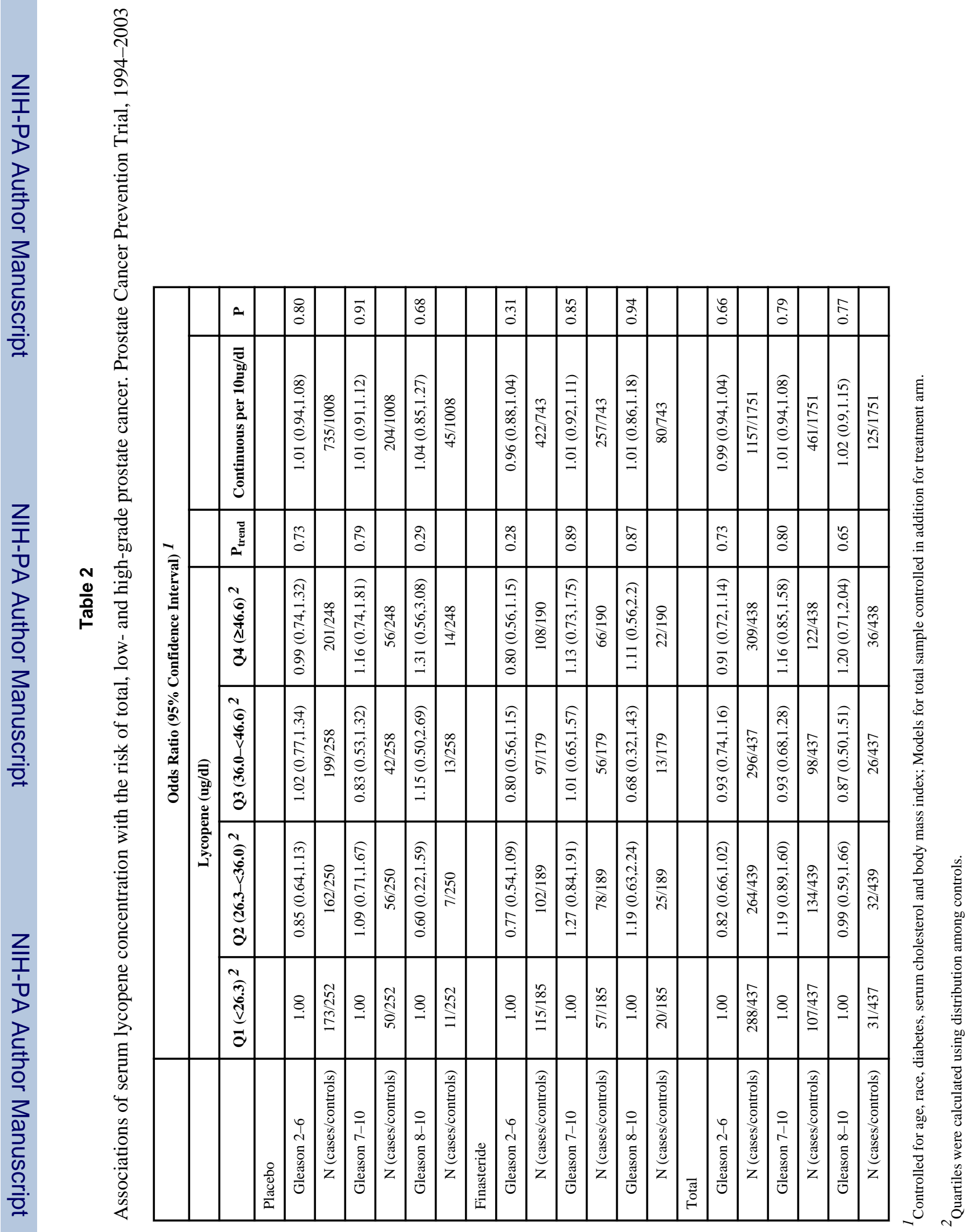




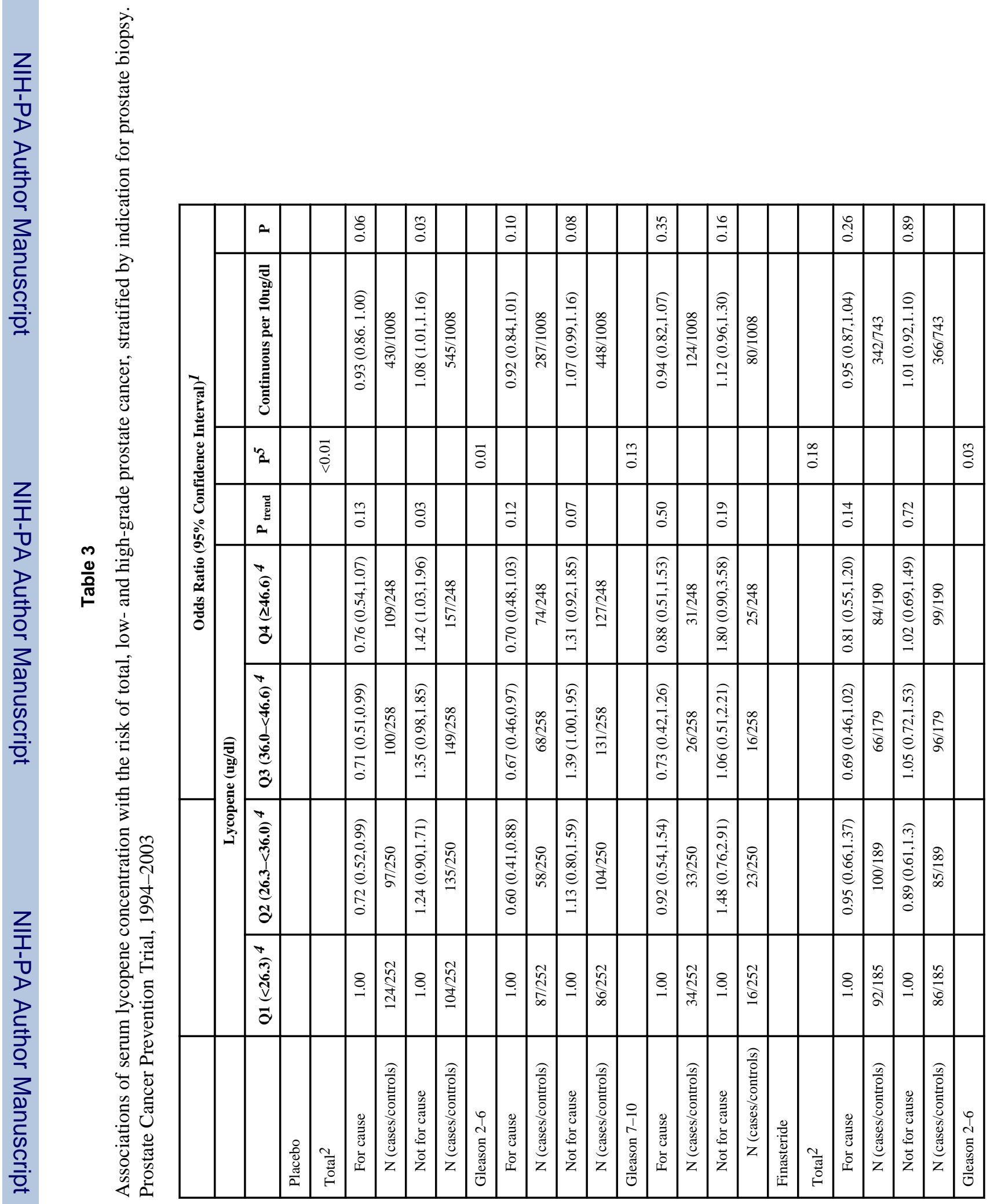




\begin{tabular}{|c|c|c|c|c|c|c|c|c|c|c|c|c|c|c|c|c|c|c|c|c|c|c|c|c|c|c|c|}
\hline \multirow{8}{*}{ 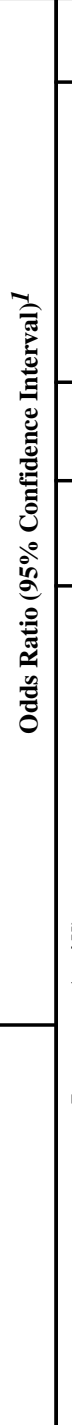 } & & $a$ & $\stackrel{+}{0}$ & & $\stackrel{n}{o}$ & & & 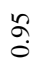 & & $\stackrel{n}{\mathfrak{o}}$ & & & & $\stackrel{0}{0}$ & & $\stackrel{\infty}{\circ}$ & & & $\stackrel{\Xi}{0}$ & & $\frac{0}{0}$ & & & $\tilde{n}$ & & กิ & \\
\hline & & 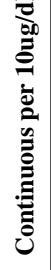 & $\begin{array}{l}\hat{\sigma} \\
\dot{0} \\
\stackrel{0}{\infty} \\
\hat{0} \\
e \\
\infty \\
\infty \\
0\end{array}$ & 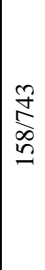 & 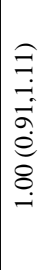 & 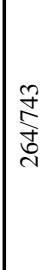 & & 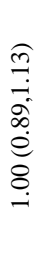 & 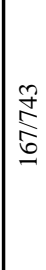 & 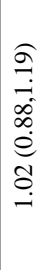 & $\frac{\stackrel{?}{f}}{\delta}$ & & & $\begin{array}{l}\widehat{\sigma} \\
\hat{\sigma} \\
\infty \\
\infty \\
\infty \\
\dot{e} \\
\dot{0} \\
\dot{0}\end{array}$ & 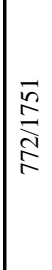 & 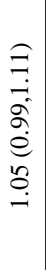 & $\begin{array}{l}\stackrel{\sqrt[n]{n}}{\triangleq} \\
\bar{\sigma}\end{array}$ & & $\begin{array}{l}\hat{o} \\
o \\
0 \\
\dot{+} \\
\infty \\
\stackrel{0}{0} \\
\bar{\sigma} \\
\dot{0}\end{array}$ & 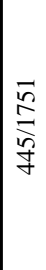 & $\begin{array}{l}0 \\
\Xi \\
0 \\
o \\
o \\
e \\
0 \\
\stackrel{0}{0}\end{array}$ & $\frac{\sqrt[n]{\Sigma}}{\stackrel{\lambda}{\sigma}}$ & & $\begin{array}{l}\widehat{o} \\
\stackrel{.}{0} \\
\stackrel{0}{0} \\
\stackrel{e}{e} \\
\stackrel{0}{0} \\
\stackrel{0}{0}\end{array}$ & 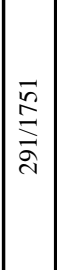 & 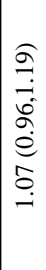 & $\frac{\sqrt{n}}{\stackrel{2}{๖}}$ \\
\hline & & 3 & & & & & ㅊ. & & & & & & $\begin{array}{l}\overrightarrow{\dot{\theta}} \\
\dot{v}\end{array}$ & & & & & $\begin{array}{l}\vec{Q} \\
\dot{\theta}\end{array}$ & & & & & $\frac{9}{0}$ & & & & \\
\hline & & ص & ö & & $\frac{2}{i}$ & & & $\begin{array}{l}\infty \\
\infty \\
0\end{array}$ & & oे & & & & ô. & & $\stackrel{0}{0}$ & & & $\stackrel{\Xi}{0}$ & & $\stackrel{0}{\circ}$ & & & $\stackrel{\infty}{n}$ & & $\stackrel{\overbrace{}}{0}$ & \\
\hline & & 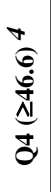 & 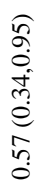 & $\underset{\infty}{\stackrel{\curvearrowright}{\curvearrowright}}$ & 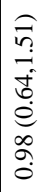 & $\underset{\gtrless}{\stackrel{2}{\Xi}}$ & & 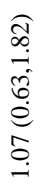 & $\underset{\ni}{\stackrel{\curvearrowright}{\Xi}}$ & 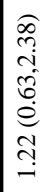 & 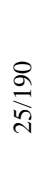 & & & 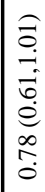 & 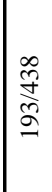 & 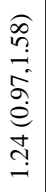 & 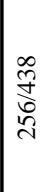 & & $\begin{array}{c}a \\
\infty \\
\infty \\
0 \\
\infty \\
+0 \\
0 \\
0 \\
0 \\
0 \\
0\end{array}$ & $\stackrel{\infty}{\stackrel{\infty}{Ð}}$ & 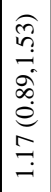 & 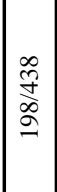 & & 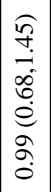 & 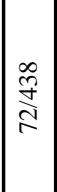 & 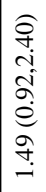 & $\begin{array}{l}\infty \\
\stackrel{\infty}{+} \\
\text { in }\end{array}$ \\
\hline & 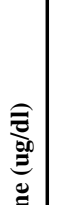 & 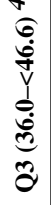 & 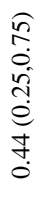 & $\stackrel{\curvearrowright}{\stackrel{2}{\curvearrowright}}$ & 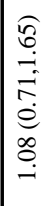 & 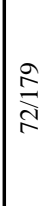 & & 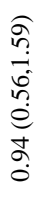 & $\underset{m}{\stackrel{2}{\curvearrowright}}$ & 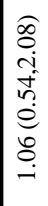 & $\underset{\text { సे }}{\stackrel{\curvearrowright}{\Xi}}$ & & & 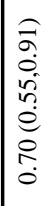 & 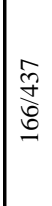 & 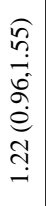 & $\frac{\hat{\tilde{y}}}{\stackrel{\tilde{y}}{\mathfrak{y}}}$ & & 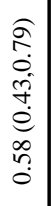 & $\frac{\tilde{\sigma}}{\stackrel{\tilde{\sigma}}{\alpha}}$ & 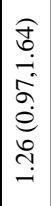 & 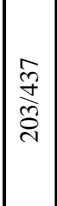 & & 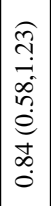 & 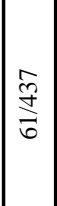 & 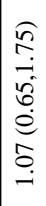 & 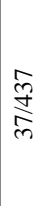 \\
\hline & 总 & 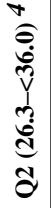 & 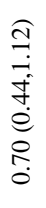 & $\stackrel{\infty}{\stackrel{\infty}{\rightleftharpoons}}$ & 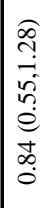 & $\underset{\text { in }}{\infty}$ & & 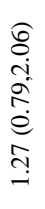 & $\sum_{i}^{\infty}$ & 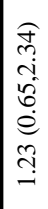 & 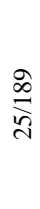 & & & 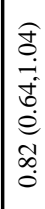 & 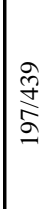 & 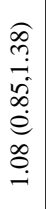 & 官 & & $\begin{array}{c}0 \\
0 \\
\infty \\
0 \\
\infty \\
0 \\
+ \\
0 \\
0 \\
0 \\
0 \\
0\end{array}$ & 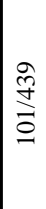 & 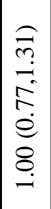 & 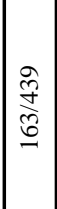 & & 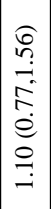 & 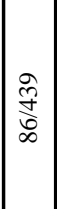 & 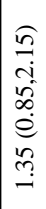 & $\frac{\underset{\sigma}{\infty}}{\stackrel{\infty}{+}}$ \\
\hline & & 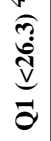 & $\underset{-}{8}$ & $\stackrel{\infty}{\infty}_{n}^{\infty}$ & $\stackrel{8}{8}$ & $\stackrel{\infty}{\infty}$ & & $\underset{8}{8}$ & $\underset{\infty}{\infty}$ & $\underset{-}{\stackrel{8}{0}}$ & $\stackrel{\infty}{\infty}$ & & & $\underset{-}{8}$ & 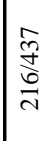 & $\underset{-}{\stackrel{-}{-}}$ & 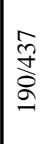 & & $\stackrel{\&}{-}$ & $\underset{\stackrel{5}{8}}{\stackrel{\delta}{g}}$ & $\underset{-}{8}$ & 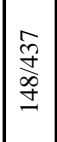 & & $\stackrel{8}{-}$ & 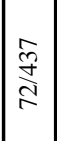 & $\stackrel{8}{8}$ & $\frac{\sqrt[m]{+}}{\sqrt[m]{n}}$ \\
\hline & & & $\begin{array}{l}0 \\
0 \\
\tilde{\Xi} \\
\tilde{0} \\
\dot{0} \\
\text { I. }\end{array}$ & 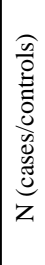 & 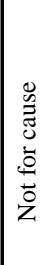 & 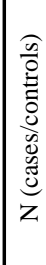 & 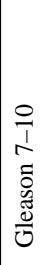 & $\begin{array}{l}0 \\
0 \\
\tilde{\Xi} \\
\vdots \\
\vdots \\
\vdots\end{array}$ & 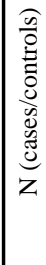 & 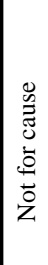 & 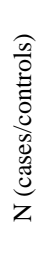 & 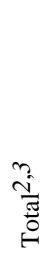 & సేّ & $\begin{array}{l}\tilde{D} \\
\tilde{\Xi} \\
\tilde{J} \\
\tilde{0} \\
\text { L }\end{array}$ & 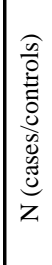 & 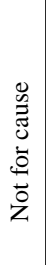 & 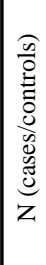 & 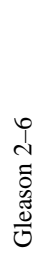 & $\begin{array}{l}0 \\
0 \\
\bar{\Xi} \\
0 \\
\vdots \\
0\end{array}$ & 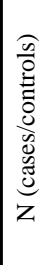 & $\begin{array}{l}0 \\
0 \\
\tilde{z} \\
0 \\
0 \\
0 \\
0 \\
z\end{array}$ & 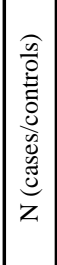 & $\begin{array}{c}0 \\
0 \\
1 \\
\tilde{I} \\
0 \\
0 \\
\tilde{d} \\
\frac{0}{0}\end{array}$ & 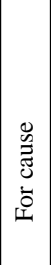 & 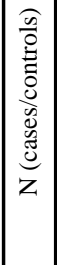 & 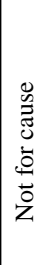 & 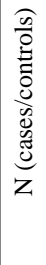 \\
\hline
\end{tabular}

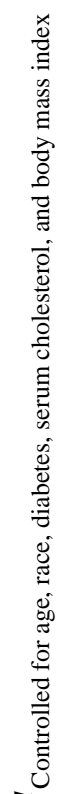




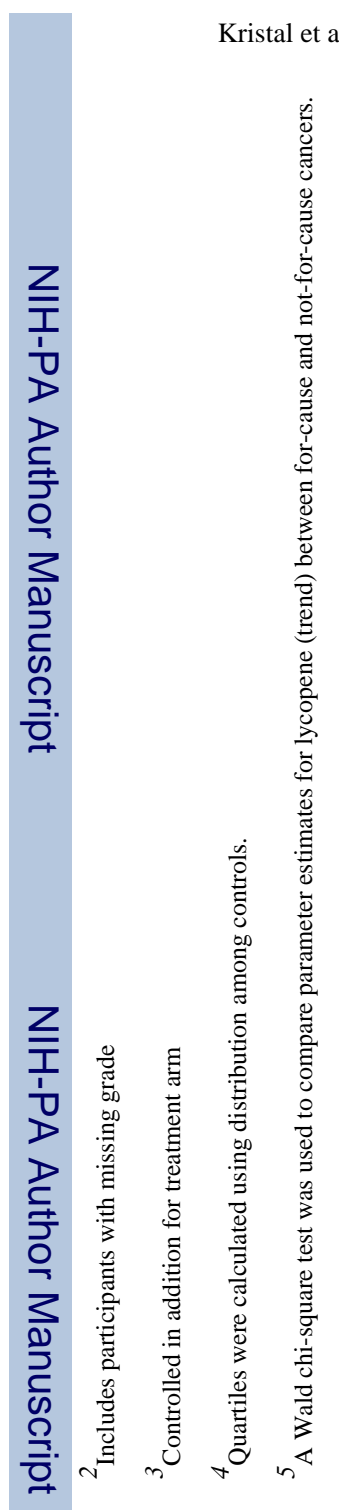

Cancer Epidemiol Biomarkers Prev. Author manuscript; available in PMC 2012 April 1. 